\title{
Remote electron beam induced current imaging of electrically active regions in $\mathrm{YBa}_{2} \mathrm{Cu}_{3} \mathrm{O}_{7-x}$ single crystals
}

\author{
C. Díaz-Guerra and J. Piqueras ${ }^{\text {a) }}$ \\ Departamento de Física de Materiales, Facultad de Físicas, Universidad Complutense, E-28040 Madrid, \\ Spain
}

(Received 15 July 1997; accepted for publication 12 September 1997)

\begin{abstract}
Remote electron beam induced current (REBIC) measurements have been carried out to investigate electrically active regions in $\mathrm{YBa}_{2} \mathrm{Cu}_{3} \mathrm{O}_{7-x}$ single crystals. Enhanced REBIC contrast, found in growth steps and other topographic features of the samples, is discussed in terms of charged oxygen-related defects. The capability of REBIC to image structural inhomogeneities caused by strain or plastic deformation in these crystals is also established. Charge carrier diffusion length has been estimated at different temperatures from REBIC linescan profiles. (C) 1997 American Institute of Physics. [S0003-6951(97)03345-7]
\end{abstract}

The investigation of the defect structure of high temperature superconductors (HTSCs) and its relation with their transport properties, is a subject of technological interest related to the development of potential device applications. Some beam injection techniques based on the scanning electron microscope (SEM), like electron beam induced voltage (EBIV) and cathodoluminescence (CL), have been applied in the past years to the characterization of these materials. Low temperature SEM-EBIV has been used to investigate transport properties of superconducting thin films and Josephson junctions. ${ }^{1,2} \mathrm{CL}$ capability to assess homogeneity of different HTSC in relation to oxygen content and distribution of oxygen-related defects, has been previously reported, e.g., Refs. 3-7. Electron beam induced current (EBIC) is another SEM-based technique which has been widely employed in the study of electrically active defects in semiconducting compounds. $^{8}$ For EBIC measurements the formation of a barrier, like a Schottky barrier or a $p-n$ junction, is necessary. When a nondestructive method is required, the use of an alternative geometry with two ohmic contacts at opposite ends of the sample is more appropriate. This technique is called remote EBIC or REBIC. ${ }^{9}$ Electron-hole pairs created by the SEM electron beam are separated by built-in electric fields associated with different charged defects and produce a current in an external circuit which, after amplification, is used to image the spatial distribution of electrically active regions in the sample. REBIC has been used in the study of the mechanisms of electronic recombination and electrical conduction of narrow gap semiconductors and semiinsulating materials ${ }^{10,11}$ but its capability to characterize HTSC has been, to our knowledge, not previously investigated. In this work, REBIC is used to study HTSC single crystals in which the presence of regions with enhanced electronic recombination has been observed by CL. ${ }^{6}$

Platelike $\mathrm{YBa}_{2} \mathrm{Cu}_{3} \mathrm{O}_{7-x}$ (YBCO) single crystals grown on top of the melt (Superconix Inc.) were investigated. The samples exhibited a Meissner transition onset at $85 \mathrm{~K}$. REBIC measurements were performed in a Hitachi S-2500 SEM. REBIC signal was detected with a Matelect ISM-5 system. Ohmic contacts, separated about $2 \mathrm{~mm}$ on the sur-

${ }^{a)}$ Electronic mail: piqueras@eucmax.sim.ucm.es faces, were provided by silver paste with gold wires. These observations were carried out at accelerating voltages ranging from 10 to $25 \mathrm{kV}$ and beam currents between $10^{-9}$ and $10^{-7} \mathrm{~A}$. Temperature was varied between 80 and $300 \mathrm{~K}$.

SEM images in the secondary electron (SE) mode reveal diverse topographic features in the crystals investigated. Some areas show rather smooth surfaces crossed by growth steps or terraces [Fig. 1(a)], while other regions show a layered structure, also a consequence of the growth process [Fig. 2(a)]. A characteristic black-white contrast appears to be associated with the steps in the corresponding REBIC images, as in both edges of the double step appearing as a vertical stripe in Figs. 1(b)-1(d) and the steps between layers in Figs. 2(b)-2(d). This contrast is known as peak and trough (PAT) ${ }^{10-12}$ and has been observed in REBIC investigations of different semiconductors. Electrically charged defects present in the material act as local potential barriers in which
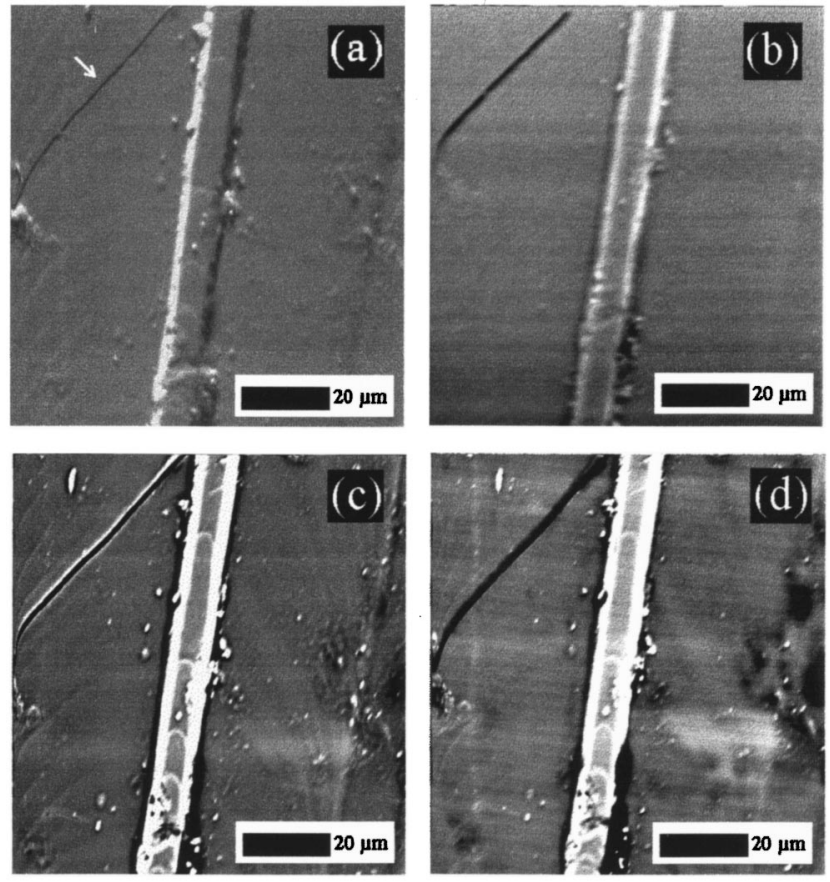

FIG. 1. (a) SE and (b)-(d) REBIC images of step structure. (b) $T=210 \mathrm{~K}$, $I_{B}=5.5 \times 10^{-8}$ A. (c) $T=80 \mathrm{~K}, I_{B}=5.5 \times 10^{-8}$ A. (d) $T=80 \mathrm{~K}, I_{B}=2.5$ $\times 10^{-8} \mathrm{~A}$. 

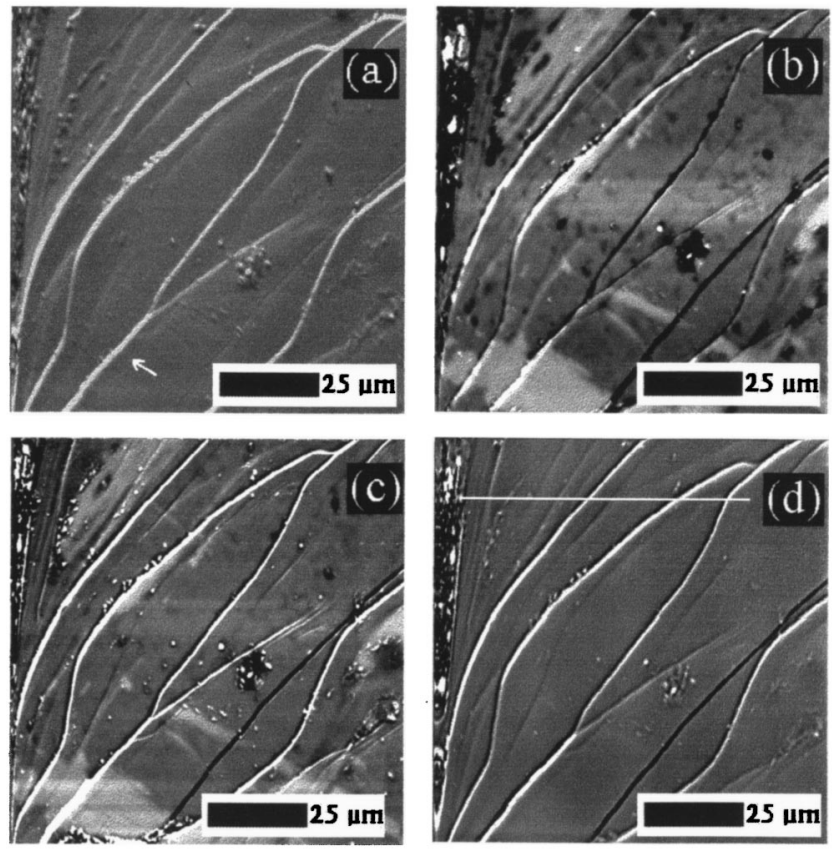

FIG. 2. Growth layers in one of the samples imaged at $80 \mathrm{~K}$ in the (a) SE and (b)-(d) REBIC modes. $I_{B}=2 \times 10^{-8} \mathrm{~A}$ (b) $I_{B}=4 \times 10^{-8} \mathrm{~A}$ (c) $I_{B}$ $=7.5 \times 10^{-8} \mathrm{~A}(\mathrm{~d})$.

carriers are separated. PAT contrast is due to opposite directions of the electric field on different sides of the defect, and can be modelled as two Schottky barriers back-to-back. ${ }^{12,13}$ The orientation of this bimodal feature is determined by the local defect geometry and the contact configuration. Another kind of contrast not related to topographic features, as the bright areas in the smooth regions between steps, can be observed under certain experimental conditions [Figs. 1(d) and 2(b)-2(c)]. Series of REBIC images of the YBCO crystals show that the observed contrasts depend both on temperature and on the excitation density. REBIC signal becomes appreciably by cooling down to near $230 \mathrm{~K}$ and increases by decreasing temperature, as comparison of Figs. 1(b) and 1(c) shows. No contrast anomaly has been observed when cooling through $T_{C}$. The dependence of REBIC contrast on the SEM beam current $\left(I_{B}\right)$ is presented in Fig. 2. The contrast not related to topographic features disappears when $I_{B}$ is higher than $5 \times 10^{-8} \mathrm{~A}$, as a comparison of the flat areas between steps of Figs. 2(c) and 2(d) shows. This contrast shows the existence of inhomogeneities in the electronic recombination properties in the samples, which agrees with previous CL observations of an inhomogeneous oxygen content distribution in the crystals. ${ }^{6}$ It was also found that PAT REBIC contrast associated with some steps could be modified by changing temperature or the beam excitation conditions. This is observed in the steps marked in the upper left corner of Fig. 1(a) and the lower left corner of Fig. 2(a). While the step of Fig. 1(a) shows black contrast at temperatures above $140 \mathrm{~K}$ or $I_{B}$ lower than $5 \times 10^{-8}$ A [Figs. 1(b) and $1(\mathrm{~d})$ ], the step marked in Fig. 2(a) shows white contrast under the same experimental conditions [Figs. 2(b) and 2(c)]. However, both exhibit PAT contrast at $80 \mathrm{~K}$ and higher beam currents [Figs. 1(c) and 2(d)]. These changes are related to the influence of temperature and excitation density on the potential barrier heights associated with the defects.

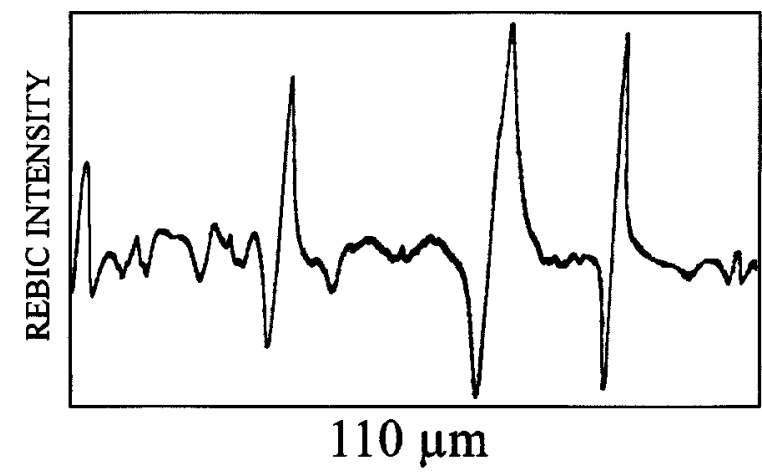

FIG. 3. REBIC linescan profile corresponding to the white line shown in Fig. 2(d).

PAT contrast can be converted into only black or only white contrast by modifying these parameters, in a way similar to the application of a bias or reverse voltage. ${ }^{11}$ When the beam injection causes a compensation of the charge state of the defect, the associated barrier disappears and the defect contrast is suppressed.

During the crystal growth process certain structural defects, mainly vacancies, are accumulated at the steps between layers. ${ }^{14}$ The influence of growth steps on the transport properties of $\mathrm{YBCO}$ and $\mathrm{Bi}_{2} \mathrm{Sr}_{2} \mathrm{CaCu}_{2} \mathrm{O}_{8+x}(\mathrm{Bi}-2212)$ single crystals has been occasionally discussed. ${ }^{15,16}$ Our previous CLSEM studies, performed on the same set of samples used in this work, revealed an enhanced $2.4 \mathrm{eV}$ CL emission in the mentioned steps. ${ }^{6}$ We obtained similar results in the CL characterization of Bi-2212 single crystals, ${ }^{7}$ in which preliminary REBIC investigations also revealed PAT contrast related to growth steps. A correlation between oxygen depletion and the mentioned $2.4 \mathrm{eV}$ emission has been established not only in $\mathrm{YBCO}^{3}$ but also in other HTSC like $\mathrm{Tl}_{2} \mathrm{Ba}_{2} \mathrm{CuO}_{6+\delta}$ (Ref. 5) and $\mathrm{Bi}-2212 .{ }^{17}$ These results suggest the possibility that charged defects, which appear associated with step structures in our REBIC micrographs, are related to areas with oxygen depletion or rearranged oxygen distributions in the crystals.

Figure 3 shows the REBIC linescan profile recorded along the line marked in Fig. 2(d). Three PAT features asymmetrical in amplitude and shape, corresponding to layer boundaries, can be observed. This asymmetry can be due to different carrier diffusion lengths $(L)$ on both sides of the defect potential barrier. Neglecting surface recombination the value of the REBIC signal can be expressed as $I \propto \exp (-x / L)$, where $x$ is the distance from the defect. ${ }^{12,18}$ Least-squares fits our experimental REBIC linescans to this exponential dependence yield $L=0.3-0.6 \mu \mathrm{m}$ at $180 \mathrm{~K}$ and $L=0.8-1.6 \mu \mathrm{m}$ at $80 \mathrm{~K}$, depending on the step considered. These measurements agree with the rough estimations of Iwabuchi et al. ${ }^{19}$ which obtained $L=0.1-0.2 \mu \mathrm{m}$ at room temperature from an EBIC image of the YBCO layer of a metal-insulator-semiconductor field effect transistor (MISFET) device.

Figure 4 shows a cracked area near the edge of one of the samples. The REBIC image reveals the existence of small sub-surface flaws [marked in Fig. 4(b)] that cannot be appreciated in the SE micrograph [Fig. 4(a)]. This image demonstrate the capability of SEM-REBIC to detect electri- 

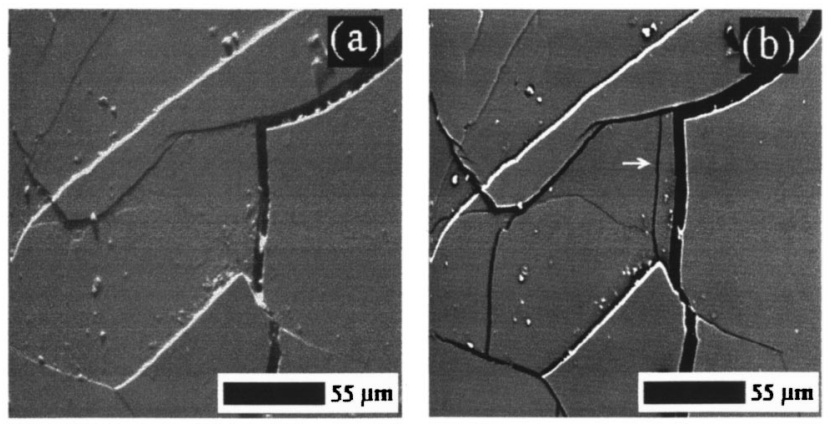

FIG. 4. (a) SE and (b) REBIC images of a cracked area in one of the crystals investigated. $T=80 \mathrm{~K} . I_{B}=7 \times 10^{-8} \mathrm{~A}$.

cally active structural defects associated with strain or plastic deformation in the YBCO crystals.

In summary, REBIC is a nondestructive suitable technique for the characterization of electrically active defects in YBCO single crystals. Charged defects accumulated at different step structures, related to oxygen deficiency by previous CL investigations, as well as structural inhomogeneities associated with plastically deformed areas, have been imaged with high resolution. An estimation of the charge carrier diffusion length at different temperatures was obtained from the experimental REBIC linescan profiles.

This work was supported by DGICYT (Project No. PB93-1256) and CICYT (Project No. MAT95-1184E).
${ }^{1}$ Y. J. Feng, Q. H. Cheng, P. H. Wu, and H. M. Liu, J. Appl. Phys. 72, 5350 (1992).

${ }^{2}$ R. Gross and D. Koelle, Rep. Prog. Phys. 57, 651 (1994) and references therein.

${ }^{3}$ P. Gómez, J. Jiménez, P. Martín, J. Piqueras, and F. Domínguez-Adame, J. Appl. Phys. 74, 6289 (1993).

${ }^{4}$ P. Gómez, J. Piqueras, M. J. Sayagués, and J. M. Gónzalez-Calbet, Solid State Commun. 96, 45 (1983).

${ }^{5}$ C. Díaz-Guerra, J. Piqueras, and C. Opagiste, Physica C 259, 121 (1996).

${ }^{6}$ C. Díaz-Guerra and J. Piqueras, J. Appl. Phys. 80, 998 (1996).

${ }^{7}$ C. Díaz-Guerra and J. Piqueras, Physica C 275, 37 (1997).

${ }^{8}$ D. B. Holt, in SEM Microcharacterization of Semiconductors, edited by D. B. Holt and D. C. Joy (Academic, New York, 1989), pp. 241-338.

${ }^{9}$ L. O. Bubulac and W. E. Tennant, Appl. Phys. Lett. 52, 1255 (1988).

${ }^{10}$ G. Panin and E. Yakimov, Semicond. Sci. Technol. 7, A150 (1992).

${ }^{11}$ D. B. Holt, B. Raza, and A. Wojcik, Mater. Sci. Eng. B 42, 14 (1996).

${ }^{12}$ E. Ziegler, W. Siegel, H. Blumtritt, and O. Breitenstein, Phys. Status Solidi 72, 593 (1982).

${ }^{13}$ G. J. Rusell, M. J. Robertson, B. Vincent, and J. Woods, J. Mater. Sci. 15, 939 (1980).

${ }^{14}$ N. Cabrera and R. V. Coleman, in The Art and Science of Growing Crystals, edited by J. J. Gilman (Wiley, New York, 1966).

${ }^{15}$ W. K. Kwok, U. Welp, K. G. Vandervoort, Y. Fang, G. W. Crabtree, and J. Z. Liu, Appl. Phys. Lett. 57, 1268 (1990).

${ }^{16}$ A. K. Pradhan, B. K. Roul, S. B. Roy, P. Chaddah, D. Kanjilal, C. Chen, and B. M. Wanklyn, Solid State Commun. 101, 367 (1997).

${ }^{17}$ P. Gómez, J. Piqueras, A. Remón, J. A. García, J. Jiménez, and A. Torres, Phys. Status Solidi A 157, 469 (1996).

${ }^{18}$ J. Palm, J. Appl. Phys. 74, 1169 (1993).

${ }^{19}$ M. Iwabuchi, T. Fuji, and T. Kobayashi, Jpn. J. Appl. Phys., Part 2 32, L491 (1993). 